\title{
Evaluation of the HIV Case-Based Surveillance System: A Pilot of the Electronic Health Record System in Mutare District, Zimbabwe, 2021
}

\author{
Kudzai Patience Takarinda1, Simon Nyadundu², Emmanuel Govha1, Notion Tafara Gombe ${ }^{3}$, \\ Tsitsi Juru ${ }^{*}$, Tshimanga Mufuta ${ }^{1}$ \\ ${ }^{1}$ Department of Primary Health Care Sciences, Global and Public Health Unit, University of Zimbabwe, Harare, Zimbabwe \\ ${ }^{2}$ Zimbabwe Ministry of Health and Child Care, Provincial Medical Directorate, Mutare, Zimbabwe \\ ${ }^{3}$ African Field Epidemiology Network, Harare, Zimbabwe \\ Email:kptakarinda@gmail.com, simonnyadundu@yahoo.com,govha86@gmail.com,ntgombe@gmail.com, \\ ^tsitsijuru@gmail.com, tshimangamufuta@gmail.com
}

How to cite this paper: Takarinda, K.P. Nyadundu, S., Govha, E., Gombe, N.T., Juru, T. and Mufuta, T. (2021) Evaluation of the HIV Case-Based Surveillance System: A Pilot of the Electronic Health Record System in Mutare District, Zimbabwe, 2021. Open Journal of Epidemiology, 11, 483-500. https://doi.org/10.4236/ojepi.2021.114039

Received: August 23, 2021

Accepted: November 19, 2021

Published: November 22, 2021

Copyright $\odot 2021$ by author(s) and Scientific Research Publishing Inc. This work is licensed under the Creative Commons Attribution International License (CC BY 4.0).

http://creativecommons.org/licenses/by/4.0/

\begin{abstract}
Background: Zimbabwe started HIV case-based surveillance in April 2017. Rapid testing for HIV recent infection was introduced into routine HIV and testing services in 2019 along with the Impilo Electronic Health Record System. For the period January-June 2020, only 1 out of 13 health facilities in Mutare district reported seven newly diagnosed HIV patients through the electronic health record system compared to 483 in the District Health Information System (DHIS-2) recorded from paper-based registers. We evaluated the case-based surveillance system attributes, usefulness and reasons for under-reporting from January-December 2020. Methods: We conducted a descriptive cross-sectional study using updated Centres for Disease Control guidelines for evaluating public health surveillance systems. Questionnaires were administered to 36 health workers involved in HIV testing services. Facility checklists were used to collect data on knowledge, system attributes and usefulness of the system. Completed HIV case-based surveillance forms were assessed for completeness. Epi Info Version 7 was used to generate frequencies, means and proportions. Results: The reasons for under-reporting of patients in the electronic health record system were lack of reporting guidelines $26 / 36(72 \%)$, limited coordination between technical staff and health facilities $24 / 36(67 \%)$ and limited competency on the Electronic health record system 22/36 (61\%). Timeliness, completeness, and validity were $88 \%, 82 \%$ and $100 \%$ respectively. The stability of the system was affected by the lack of standard operating procedures during system interruptions. Overall representativeness was $45 \%$ despite increasing from 3/226 (1\%) to 224/303 (73\%) between Quarter-1 and
\end{abstract}


Quarter-4 of 2020. Acceptability was 100\% due to reduced paperwork and the ability to generate simple reports. The information generated was used to identify new infection hotspots 28/36 (78\%). Conclusion: The HIV cases based surveillance system was timely, acceptable with good data quality. Representativeness was poor due to limited competency on the electronic health record system. As a result, health workers received further training.

\section{Keywords}

HIV Case-Based Surveillance, Recency Testing, Electronic Health Record System, Mutare, Zimbabwe

\section{Introduction}

HIV remains a major global public health challenge with an estimated 38 million people living with HIV worldwide [1]. Approximately, two-thirds of all new HIV infections originate from the African region globally and 68\% of HIV-infected persons live in sub-Saharan Africa [2]. Zimbabwe has one of the highest HIV prevalence in sub-Saharan Africa at $12.8 \%$, with an estimated 1.4 million People Living with HIV (PLHIV) and 40,000 new HIV cases recorded in Zimbabwe in 2019 [3].

\subsection{Overview of the HIV Case-Based Surveillance System}

The World Health Organization (WHO) launched the consolidated guidelines on person-centered HIV patient monitoring and case surveillance in 2017 [4]. HIV Case-Based Surveillance (CBS) is the systematic, continuous reporting and analysis of routinely generated longitudinal individual-level (single case) demographic and health event (sentinel) data about people living with HIV [5]. By 2019, 20/39 (51\%) United States PEPFAR supported countries had implemented HIV CBS [6]. The implementation of HIV case-based surveillance systems is influenced by several factors including country policies and guidance on mandatory reporting, strong political support as well as local technical and programming expertise, which are critical for successful implementation [7].

\subsection{Objectives of HIV Case-Based Surveillance System}

Zimbabwe implemented HIV CBS in April 2017 in a phased approach in two districts, Mutare and Umzingwane [8]. The objective of the HIV CBS is to identify and describe geographic locations associated with newly diagnosed cases including recent infections and inform geographic prioritization of HIV prevention interventions [9]. The system also aims to monitor and describe epidemiological trends in newly diagnosed HIV cases by demographics, behavior, mode of transmission and recency of HIV infection. Additionally, the system aims to monitor and describe trends of clinical status (WHO Stage, initial CD4 and other opportunistic infections) at the point of diagnosis [8]. 


\subsection{Flow of Data in the HIV CBS System in Zimbabwe}

At the health facility level, HIV case reports are generated at the point of service delivery such as the hospital outpatient department or inpatient department, Family and Child Health unit (FCH) and Voluntary Male Medical Circumcision unit (VMMC). Health facilities that use the electronic-first EHR system capture all related surveillance data elements into the system as the client goes through the clinic service flow. At health facilities that use an electronic-last EHR system, all surveillance data elements are recorded on a single HIV case report form. The data from this case report form is then transferred into the EHR system using an on-site mobile device. Data from both e-first and e-last clinic-based EHR systems are merged in the national shared health record component through an interoperability layer. A command to de-duplicate all health records across clinics using national identification number, passport number, or driver license number is given at the national level. Deduplicated, and anonymized individual health records are transmitted to the National Ministry of Health and Child Care data warehouse from the shared health record component [10] (Figure 1).

The Rapid Test for HIV Recent Infection (RTRI) was introduced in routine HIV and Testing Services (HTS) in September 2019 allowing access to recency testing for persons with newly diagnosed HIV at all facilities and community-based testing sites in the country [11]. The adoption of HIV incidence assays in the national HIV testing algorithm allows person, place and time to be described for all new HIV infections as part of high throughput case-finding strategies in identified clusters [12].

Thirteen health facilities in Mutare District in eastern Zimbabwe piloted the

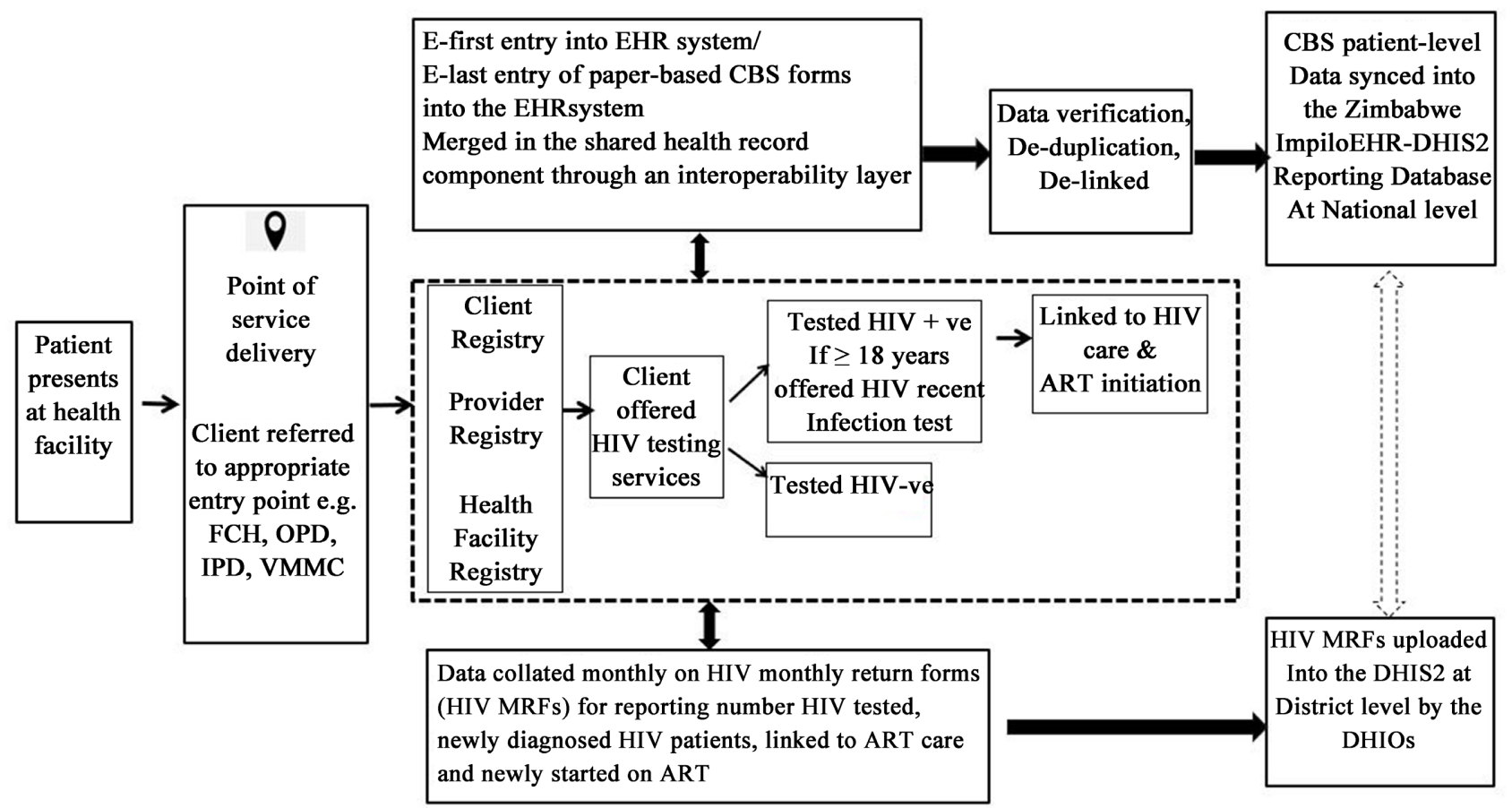

Figure 1. The HIV case-based surveillance information flow chart in Zimbabwe. 
Impilo Electronic Health Record (EHR) system in 2019 as part of efforts to reduce inefficiencies associated with paper data collection and reporting and to improve patient care. At these facilities, patient-level data for all newly diagnosed HIV patients collected through CBS forms, should be entered and reported through the Zimbabwe Impilo electronic health record system. Reported figures should tally with the monthly aggregate number of newly diagnosed HIV patients reported through the DHIS2 system which is collated from the HIV monthly return forms at the health facility level where the primary data source is HIV Testing Services (HTS) registers. For the period January-June 2020, only 1 out of 13 EHR implementing health facilities in Mutare district reported seven newly diagnosed HIV patients out of a total of 483 .

The HIV-CBS system with recency testing at EHR implementing facilities in Mutare district is prone to under-reporting of newly diagnosed HIV cases which may results in poor data that is not representative of the population. The resulting irrelevant and inaccurate policy conclusions will ultimately undermine continued efforts to achieve epidemic control of the HIV/AIDS pandemic in line with the Joint United Nations Programme on HIV/AIDS (UNAIDS) 2030 targets. We evaluated the HIV CBS system with recency testing in Mutare district to determine the reasons for under-reporting of newly diagnosed HIV cases through the EHR system, assessed the attributes of the HIV CBS system, determined the usefulness of the system and assessed health workers' knowledge of the HIV CBS system in the paper.

\section{Methods}

\subsection{Study Design}

We conducted a descriptive cross sectional study using the updated Centres for Disease Control (CDC) guidelines for evaluating public health surveillance systems [13].

\subsection{Study Setting}

We conducted the study in Mutare district which is one of the seven districts in Manicaland province located in eastern Zimbabwe. The Zimbabwe National Statistics Agency (ZIMSTAT) District-population report projected a population of 216,511 in Mutare urban and 313,645 in Mutare rural for 2020 [14]. According to DHIS-2, the district has a total of 44 health facilities. The Government of Zimbabwe owns $39 \%$ of all health facilities, while the majority, (52\%) are run by the rural district authorities. Health facilities comprising of mission hospitals and clinics constitute 7\%, while the Wattle Company owns 2\%. Victoria Chitepo Provincial Hospital is in Mutare district and is the only tertiary hospital in Manicaland province. All health facilities offer HIV testing and counseling services, however, 13 health facilities had implemented the electronic health record at the time of the study. The main economic activities in the area are citrus farming, forestry and mining. 


\subsection{Study Population}

We interviewed health care workers involved in the HIV CBS system and HIV testing and counseling services as primary respondents. The District Medical Officer, matron, sister in charge, District health information officer, Provincial health information officer and four community health nurses were also interviewed as key informants.

\subsection{Sample Size Calculations}

To assess for completeness of HIV CBS forms, we calculated a minimum sample size of 80 HIVCBS reporting forms using Dobson's formula for calculating sample size for a single proportion:

$$
\left(Z \alpha^{2}(p)(1-p)\right) / c^{2}
$$

where:

$$
\begin{aligned}
& Z=Z \text { statistic value i.e. } 1.96 \text { for a } 95 \% \text { confidence interval; } \\
& p=\text { proportion of HIV CBS reporting forms with } 76 \% \text { completeness; } \\
& c=10 \% \text { precision. }
\end{aligned}
$$

Assuming that completeness of HIV CBS reporting forms was $76 \%$ complete and given a $95 \%$ confidence interval, $10 \%$ level of precision and $10 \%$ non response rate based on a study by Hall et al., 2006 [15].

\subsection{Sampling Techniques}

\subsubsection{Sampling of Health Facilities}

We purposively selected all 13 health facilities that had implemented both HIV CBS and the EHR system into the study. These were Sakubva district hospital, Chikwariro Clinic, Chitakatira Rural District Council Clinic, Dangamvura Polyclinic, Marange Rural Health Clinic, Nyagundi Rural Health Clinic, Odzi Clinic, Sakubva Clinic, St. Andrews Hospital, St. Joseph's Mission Hospital, St. Werburgh's Clinic, Zimunya Clinic and Zvipiripiri Rural Health Clinic.

\subsubsection{Sampling of Primary Participants}

We enrolled all $(\mathrm{N}=36)$ health workers involved in HIV testing and counseling and data entry found on duty at the health facilities during the study.

\subsubsection{Sampling of HIV Case-Based Surveillance Forms}

We used the random sample formula in Microsoft excel "=RAND()" to sample 7 forms from each of the 13 health facilities included in the study. We created a line-list of all identified reporting forms in an Excel spreadsheet using the monthly sequential number as obtained from the HIV testing services (HTS) register. We sampled 91 paper-based HIV CBS forms for the period between January-December 2020.

\subsubsection{Sampling of Key Informants}

We purposively enrolled 8 key informants into the study. 


\subsection{Data Collection}

We used interviewer-administered questionnaires to collect the demographic characteristics of healthcare workers participating in the study and information on the reasons for under-reporting of newly diagnosed HIV patients in the EHR system from healthcare workers and key informants as well as assessing the system attributes.

Health Worker Knowledge on the HIV CBS System with Recency Testing We used interviewer-administered questionnaires to assess the knowledge of the health workers on the HIV CBS system with recency testing using the following variables: ability to accurately define a case, ability to describe the purpose of HIV CBS system, ability to state what information is collected from a case in the HIV CBS system, ability to accurately state what sentinel events are reportable, and ability to describe the steps taken after an HIV case is diagnosed. We used a 3 point Likert scale used to assess the quality of health worker knowledge level as described by Brown in 2010 [16]. Health workers who answered less than or equal to two (2) questions correctly out of 5 were classified as having poor knowledge levels and those that got three (3) out of five had fair knowledge level. Participants that answered greater than or equal to four (4) questions correctly were classified as having good knowledge level.

\subsection{Evaluation of HIV CBS System Attributes}

\subsubsection{Representativeness}

Representativeness measures the degree to which the CBS system is capturing all cases and sentinel events and the degree to which cases in the surveillance system are representative of all cases in the population. We assessed representativeness by examining proportion of HIV cases reported in the EHR system compared to HIV cases recorded in the DHIS-2 using monthly return forms. We measured representativeness as the: 1) proportion of healthcare facilities in the district (private or public) that submit case reports to the health facility; and 2) proportion of HIV cases diagnosed in a quarter that were reported in the DHIS-2 and the EHR system.

\subsubsection{Timeliness}

Timeliness describes the amount of time between steps in the case reporting and data management process. We determined timeliness by assessing the lag time between diagnosis and entering of the case report in the EHR system.

\subsubsection{Data Quality}

Data quality reflects the completeness and validity of the data recorded in the HIV CBS system. Completeness describes the proportion of case reports that contain an expected variable or variables. We assessed completeness of the HIV CBS system by calculating the proportion of case reports that had all case defining variables complete. The "unknown" or "blank" responses to items on the case report forms were deemed as incomplete if applicable to the HIV case. We also 
assessed data quality by the number of trainings, supervision and data quality assurance reviews conducted. Validity describes the degree to which the CBS data "makes sense," or whether the data falls within the range of possible or expected values. We assessed validity using year of birth which should be four digits between the range of 1940 and 2020.

\subsubsection{Simplicity}

Simplicity refers to ease of operation, structure, and integration of incidence surveillance with routine HIV surveillance. We assessed simplicity of the HIV CBS system with recency testing using the proportion of health workers who found the completion of paper-based and electronic forms as easy and not time-consuming (average time taken to complete these forms) and staff training requirements. We assessed competence of the surveillance system using the following criteria: ability to login into the Impilo EHR DHIS-2 system, create an HIV electronic health record, retrieve a client's electronic health record, edit or update an electronic health record, delete an electronic health record and generate a report.

\subsubsection{Acceptability}

Acceptability reflects the willingness of the healthcare workers to participate in HIV case-based surveillance. We assessed acceptability through interviews with health workers. We calculated the proportion of healthcare workers who were willing to continue participating in the system and rely on data from it. We objectively assessed acceptability of the HIV CBS system using completeness, quality of data, timeliness, availability of minutes of meetings and feedback reports.

\subsubsection{Flexibility}

A flexible surveillance system can adapt to changing information needs or operating conditions with little additional time, personnel, or allocated funds. We assessed the flexibility of the HIV CBS system by observing how the system has responded to new information and technology requirements such as HIV recency testing and the EHR system.

\subsubsection{Stability}

Stability refers to the reliability and availability of the HIV case-based surveillance system. We assessed stability of the surveillance system by ascertaining the presence of dedicated staff for HIV CBS activities and the level of interruption of the system due to inadequate human resources, EHR system down-time due to electricity outages, availability of case report forms, dedicated computers and tablets to enter the case reports using a checklist.

\subsubsection{HIV CBD System Usefulness}

We assessed the usefulness of the HIV case-based surveillance system by asking respondents if the collected data was analyzed, the data uses and any reports or graphs generated from the data. The respondents were also asked about the public health actions carried out or made based on the findings from data collected 
by the surveillance system. We also assessed the availability of minutes of meetings held on the surveillance system and any actions taken to validate the usefulness of the system.

\subsection{Data Analysis}

We used Epi Info TM version 7.2.4 for data entry and analysis. Descriptive statistics were used to analyze quantitative the data and was presented as medians, interquartile ranges, frequencies and proportions. A 3-point Likert scale was used to rate the respondent's knowledge.

\subsection{Ethical Considerations}

We obtained permission to conduct the study from the Provincial Medical Director (PMD) Manicaland Province, Health Director Mutare City and District Medical Officer (DMO) Mutare District and Health Studies Office. We obtained written informed consent from all respondents. We did not use participants' names or addresses during the study. Written informed consent was obtained from all respondents. We observed COVID-19 infection, prevention and control practices during the interviews.

\section{Results}

\subsection{Demographic Characteristics of Health Workers Involved in HIV CBS Activities at EHR Implementing Facilities in Mutare District, 2021}

We recruited 36 healthcare workers into the study, the majority being female 25/36 (69\%) and primary care counselors $17 / 36(47 \%)$. The median age of study participants was 41.5 years [Inter-quartile range (IQR), 35 - 45.5] and the median years in service was 7 years (IQR, 4 - 12). The responsible authorities for the 13 EHR implementing health facilities were as follows: government (4), mission (4), rural district council (3) and city council (2) (Table 1).

The major reasons reported by health workers for under-reporting of newly diagnosed HIV cases through the EHR system were lack of reporting guidelines 26/36 (72\%), limited coordination between the EHR system technical staff and the health facilities $24 / 36$ (67\%), insufficient health worker knowledge of the EHR system 22/36 (61\%). Lack of feedback from higher levels on reporting requirements and performance, 19/36 (53\%) and use of parallel systems 17/36 (47\%) were also cited as reasons for under-reporting (Table 2).

\subsection{Health Worker Knowledge of the HIV Case-Based Surveillance System at EHR Implementing Facilities in Mutare District, 2021}

Of the 36 health workers assessed on awareness and understanding of the HIV CBS system, 29 (81\%) were able to accurately define an HIV case while 31 (86\%) were able to describe the objectives of HIV CBS system. Ninety-four percent (34/36) knew which information was collected for HIV case notification and 
Table 1. Demographic characteristics of health workers involved in HIV CBS activities at EHR implementing facilities in Mutare district, 2021.

\begin{tabular}{|c|c|c|c|}
\hline Variable & Categories & Frequency $n=36$ & Percentage \\
\hline \multirow[t]{2}{*}{ Sex } & Female & 25 & 69 \\
\hline & Male & 11 & 31 \\
\hline \multirow[t]{4}{*}{ Designation } & Primary care counselor & 17 & 47 \\
\hline & RGN & 7 & 19 \\
\hline & PCN & 7 & 19 \\
\hline & Data entry clerks & 5 & 14 \\
\hline Years in service & $\begin{array}{l}\text { Median years in service } 7 \\
\qquad \mathrm{Q}_{1}=4 ; \mathrm{Q}_{3}=12\end{array}$ & & \\
\hline \multirow{6}{*}{ Age group (years) } & $25-34$ & 9 & 25 \\
\hline & $35-44$ & 17 & 47 \\
\hline & $45-54$ & 8 & 22 \\
\hline & $55-64$ & 2 & 6 \\
\hline & Median age 41.5 & & \\
\hline & $\mathrm{Q}_{1}=35.0 ; \mathrm{Q}_{3}=45.5$ & & \\
\hline
\end{tabular}

Table 2. Reasons for under reporting of newly diagnosed HIV cases through the Impilo EHR system at health facilities in Mutare district, 2021.

\begin{tabular}{lcc}
\hline Reason for under-reporting & Frequency $\mathbf{n}=\mathbf{3 6}$ & Percentage \\
\hline No reporting guidelines & 26 & 72 \\
Limited coordination between units & 24 & 67 \\
Inadequate EHR system competence & 22 & 61 \\
No feedback from higher levels & 19 & 53 \\
Use of parallel systems for reporting cases & 17 & 47 \\
Work overload/short staffed & 15 & 42 \\
Poorly designed interface & 15 & 42 \\
System downtime related challenges & 15 & 42 \\
Too many data sources required to fill the form & 11 & 31 \\
Electricity power outages & 11 & 31 \\
High staff turn over & 10 & 28 \\
Limited or non-existent incentives & 9 & 25 \\
Poor network connectivity & 2 & 6
\end{tabular}

29/36 (81\%) could accurately state reportable sentinel events. Actions taken after an HIV case is diagnosed were accurately described by $32 / 36$ (89\%) of healthcare workers. Using a 3-point Likert scale, 29/36 (55\%) of healthcare workers had good knowledge, while 5/36 (14\%) had fair knowledge and 2/36 (36\%) had poor knowledge of the HIV CBS system (Table 3). 
Table 3. Health worker knowledge levels of the HIV case-based surveillance system with recency testing at EHR implementing facilities in Mutare district, 2021.

\section{Variable}

Health worker knowledge levels of HIV CBS

Health workers who knew at least two $(\geq 2 / 3)$ definitions of an HIV case

Health workers who knew at least two ( $\geq 2 / 4)$ objectives of the HIV CBS system

Health workers who knew at least eight $(\geq 8 / 16)$ patient information variables collected on a HIV case

Health workers who knew at least four $(\geq 4 / 8)$ reportable sentinel events

Health workers who knew at least $2(\geq 2 / 4)$ actions to be taken when an HIV case has been identified

Overall health worker knowledge levels of the HIV CBS system

\begin{tabular}{cccc} 
Good & $(4-5$ correct $)$ & 29 & 80 \\
Fair & $(3$ correct $)$ & 5 & 14 \\
Poor & $(1-2$ correct $)$ & 2 & 6 \\
\hline
\end{tabular}

\subsection{The HIV CBS System Attributes at EHR Implementing Facilities in Mutare District}

\subsubsection{Representativeness}

Compared to the DHIS-2 system, 3/226 (1\%) of HIV cases were notified through the EHR sys-tem from January-March 2020 and increased to 224/303 (73\%) by October-December 2020. Overall representativeness was $45 \%$. There were no private facilities reporting cases through HIV CBS system, however, 27/36 (75\%) of healthcare workers reported that there were several development partner organizations who performed community testing, outreach activities as well as HIV self-test kit distribution and reported HIV cases to the facilities through the HIV CBS system.

\subsubsection{Data Quality}

The overall completeness of the 91 HIV CBS forms assessed was $82 \%$. Data completeness ranged from as high as $95 \%$ for demographic data to $88 \%$ for HIV diagnosis test information to as low as $52 \%$ for facility information. Validity was at $100 \%$ as all 91 HIV CBS assessed had patients' age verified by comparing age of patient with calculated age based on date of birth. Thirty-three healthcare workers (92\%) reported that they had received a supervisory visit from the higher level. The median reported number of supervisory visits was 6 (IQR, $3-8$ ) (Table 4).

\subsubsection{Timeliness}

Out of the 36 health workers interviewed, 11 (31\%) had completely migrated from paper-based notification to electronic-first notification in the EHR system compared to 25 (69\%) who were still using both notification systems. At facilities 
Table 4. Data quality of the HIV Case-based surveillance system at EHR implementing facilities in Mutare district, 2021.

\begin{tabular}{|c|c|c|c|}
\hline Variable & $\begin{array}{l}\text { Frequency } \\
\qquad \mathrm{N}=91\end{array}$ & Percentage & $\begin{array}{c}\text { Completeness } \\
(\%)\end{array}$ \\
\hline Section A: demographic Information & 91 & 100 & 95 \\
\hline Section B: HIV diagnosis test information & 90 & 99 & 88 \\
\hline \multicolumn{4}{|l|}{ Section C: client aged 0 - 24 month } \\
\hline Infant & 6 & 7 & 83 \\
\hline Mother & 6 & 7 & 78 \\
\hline \multicolumn{4}{|l|}{ Section D: clients Aged $\geq 25$ months } \\
\hline Transmission risk & 91 & 100 & 89 \\
\hline $\begin{array}{l}\text { Rapid test for HIV recent infection (client aged } \\
15 \text { years and above) }\end{array}$ & 80 & 88 & 81 \\
\hline Assessment of WHO clinical staging at notification & 78 & 86 & 74 \\
\hline ART initiation & 82 & 90 & 89 \\
\hline $\begin{array}{l}\text { Information on facility entering notification } \\
\text { form into the EHR/EPMS }\end{array}$ & 72 & 78 & 52 \\
\hline Validity & 91 & 100 & \\
\hline Supervisory visits from higher levels & \multicolumn{2}{|c|}{ Median $6 \mathrm{Q}_{1}=3$} & $\mathrm{Q}_{3}=8$ \\
\hline
\end{tabular}

using both the paper-based and electronic sys-tem, 22/25 (88\%) healthcare workers entered the HIV CBS forms daily. Data transmission from facility- to national-level was automatic (real time) once entered as reported by $32 / 36(89 \%)$ of the healthcare workers (Table 5).

\subsubsection{Simplicity}

Out of the 36 health workers interviewed, 30 (94\%) reported that HIV CBS forms were not difficult to complete. The median time taken to complete one form was 11.0 minutes (IQR, 7.5 - 14.5) compared to 16 minutes (IQR, 7.5 - 25.5) when completing a case notification form in the EHR system. Competency in the use of the EHR system ranged from 35/36 (97\%) health workers being able to login into the EHR system, to $11 / 36$ (31\%) being able to edit or update an electronic health record (Table 5).

\subsubsection{Acceptability}

All $36(100 \%)$ healthcare workers reported their willingness to continue participating in the HIV CBS system and had previously notified a case while 34/36 (94\%) reported that they were part of a team that analyzed and utilized HIV CBS data (Table 5).

\subsubsection{Flexibility}

New technology such as mobile devices could be integrated with ease as reported by $34 / 36$ (94\%) of health workers while (32/36) (91\%) health workers mentioned 
Table 5. Timeliness, simplicity, flexibility, acceptability and usefulness of the HIV CBS system at EHR implementing facilities in Mutare district, 2021.

\begin{tabular}{llc}
\hline Variable & Frequency & Percentage \\
\hline Timeliness of the HIV CBS system & & \\
\hline Migration status from paper-based HIV CBS forms to EHR $(\mathrm{n}=36)$ & 11 & 31 \\
Completely migrated to EHR & 25 & 69 \\
Still using both CBS forms \& EHR & 22 & 88 \\
Time period taken for entry of paper-based HIV CBS forms into the Impilo EHR DHIS-2 system (n = \\
$25)$ & 2 & 8 \\
Daily & 1 & 4 \\
Weekly & & 89 \\
Monthly & 32 & 11 \\
Timely transmission of CBS data from facility to national level & 4 & \\
Yes & &
\end{tabular}

Simplicity of the CBS system

Login into the Impilo EHR DHIS-2 system $\quad 35$

Create an HIV electronic health record $\quad 26 \quad 72$

$\begin{array}{llll}\text { Generate a report } & 15 & 42\end{array}$

Edit or update an electronic health record $\quad 11 \quad 31$

Delete an electronic health record. $\quad 0 \quad 0$

Median time taken to complete a case notification form in EHR system Median $16 \min \left(\mathrm{Q}_{1}=10 ; \mathrm{Q}_{3}=\right.$ 25.5)

Median time taken to complete HIV CBS form

Median $11 \min \left(\mathrm{Q}_{1}=7.5 ; \mathrm{Q}_{3}=\right.$ 14.5)

\section{Acceptability of the CBS system}

Health workers willing to participate in the system

$36 \quad 100$

Health workers who notified HIV cases

$36 \quad 100$

Health workers able to do data analysis and use

3494

Health workers who notified a case in the EHR system

32

Health workers who completed an HIV CBS case notification form

30

83

\section{Flexibility of the HIV CBS system}

Ease of introducing new technology

$34 \quad 94$

Ease of integrating with other systems

3291

Ease of integrating new information requirements

$29 \quad 83$

More staff required to operate the system

$16 \quad 44$

New demands when using the system

6

19




\section{Continued}

\section{Usefulness of the HIV CBS system}

Held meeting or discussion on HIV CBS data

Data analysis done at facility level

Public Health action taken

Generate reports or graphs from HIV CBS data

Use of analyzed HIV CBS data

HIV test kits supply management

Drug supply chain management

Identify hotspots for new infections for community interventions

Identify risky behaviors for communication strategies
83

$28 \quad 78$

26

14

31

30

28

25
78

72

39

83

78

69

that the HIV CBS system could be integrated with other health information systems. Mentioned systems included DHIS-2, the electronic Patient Monitoring System (ePMS) and the EHR systems. However, 16/36 (44\%) mentioned need for more staff to operate the system. Six (19\%) healthcare workers reported that there were new demands to the HIV CBS system with recency testing involving use of electronic mobile devices which required more time to operate compared to the paper-based HIV CBS forms.

\subsubsection{Stability}

Healthcare workers dedicated towards HIV CBS activities constituted 27/36 (75\%) of respondents. Out of the 13 facilities assessed, 4 facilities did not have a generator, 3 facilities had non-functioning generators and needed repairs and 3 health facilities did not have solar power which affected the operation of the EHR system. We found that challenges were associated with fuel shortages for generators. Four facilities did not have WHO clinical staging of HIV disease guidelines. Reporting of daily and weekly statistics through a WhatsApp group platform required airtime disbursements which were not available.

\subsubsection{Usefulness of the HIV CBS System}

The majority of healthcare workers, 30/36 (83\%) reported that either a team of staff or a quality improvement committee would meet and discuss HIV CBS data while $28 / 36$ (78\%) reported that data was analyzed and utilized at their facilities. Data utilization included HIV test kits supply management as reported by $31 / 36$ (86\%) health workers and drug supply chain management as reported by $30 / 36$ $(83 \%)$ health workers. Data was also used to identify new infections hotspots for community interventions $28 / 36$ (78\%) and to identify risky behavior for communication strategies $25 / 36$ (69\%) (Table 5).

\section{Discussion}

We evaluated the HIV CBS system with recency testing to assess if the objectives 
of the system are being met, its usefulness and the reasons for under-reporting of newly diagnosed patients in the EHR system. We found that the major reason for under-reporting of HIV cases through the Impilo EHR DHIS-2 system was the lack of reporting guidelines. Health workers were not aware that they had to submit HIV CBS forms to data entry clerks for entry into the EHR system while the data entry clerks were not confident to use the EHR system. This is consistent with findings by Turnberg et al., 2010, who found that health workers were not aware of reporting guidelines for notifiable infectious diseases within their institutions resulting in underreporting of cases [17]. However, Gelsuga et al., 2017 identified user resistance, lack of training and lack of awareness of the EHR system or its importance as barriers to implementation [18].

Educating health workers about reporting requirements reduces their perception of HIV case re-porting as burdensome. Other reasons for under-reporting of HIV cases through the Impilo EHR DHIS-2 system included lack of coordination between units consistent with findings by Nsubuga et al., 2020 who found poor co-ordination between units and lack of feedback from central levels as reasons for poor performance of the system [18]. The role of feedback cannot be over-emphasized in coordinating surveillance activities, increasing awareness and reinforcing the importance of participating in the HIV CBS system

The system was not stable as electricity power outages and software upgrades affected the system in some facilities. System downtimes are unavoidable therefore, it is essential to have standard operating procedures to ensure continuity of care and system downtime recovery. An extensive back-up plan was not in place as the HIV CBS forms which may be used as backup paper based records were not available at four facilities. Seven facilities lacked functional generators for power back-up and facilities that had functional generators faced fuel shortages. Our findings were consistent with Nsubuga et al., 2020 who found that some of the challenges in the HIV CBS system were electricity outages and a lack of supplies to operate the system [19].

We found that the overall representativeness of the HIV CBS system to be poor. The availability of electronic mobile devices to health workers from August 2020 facilitated backdated entry of HIV CBS forms which resulted in improved representativeness by the end of 2020. These findings are in conflict with findings by Gortakowski et al., 2010 who found that the system accurately represented the HIV epidemic in New York City [20]. No private facilities participated in the system, however, distribution of community self-testing kits by partner agencies facilitated the inclusion of individuals irrespective of age, sex and locality. This was consistent with findings by Okeafor et al. in Rivers State Nigeria who found that representativeness was limited by non-inclusion of private health facilities [21].

We found that the timeliness of the HIV CBS system was $88 \%$ as HIV CBS forms were entered daily by the majority of healthcare workers and there was real time automatic transmission of data to the national level. This is consistent with findings by Ezeudu et al., 2016 who also found the system to be timely in 
Enugu State, Nigeria [22]. Data quality was measured by completeness and validity. We found the data quality of the HIV CBS system to be good. Completeness was high for patient demographic information, however it was low for WHO clinical staging and facility information. These findings are consistent with findings by Naqibullah et al., 2020 who found that demographic variables had a high level of completeness but the completeness of clinical (screening, diagnosis and treatment) variables were lower in Afghanistan [23]. An evaluation of the United States HIV/AIDS surveillance system by Ngugi et al., 2019 concluded that data quality was low because it was not granular enough to differentiate complex transmission risks that result from cultural and ethnic diversity [24]. This reinforces the importance of HIV CBS as these data quality shortcomings affect the usefulness of data for programming.

We found the HIV CBS to be simple when using the HIV CBS forms. However, notification of HIV cases through the EHR system was not simple as the majority of healthcare workers could only login and create an electronic health record which ultimately required more time than when using the paper based HIV CBS form. Several gaps were observed in terms of retrieval and editing of patient records, generation of reports and analysis of results. This is consistent with findings by Sukums et al., 2014 who concluded that given the low levels of computer knowledge among rural health workers in Africa, it is important to provide adequate training and support to ensure the successful uptake of electronic systems at primary health facilities in Burkina Faso, Ghana, and Tanzania [25]. Training ensures that users are competent and comfortable with the use of the new EHR system and reassessment status should be ascertained to evaluate training gaps.

The system was found to be flexible consistent with findings by Okeafor et al. who found the HIV CBS system to be flexible in Rivers State Nigeria [20]. New technology such as the use of electronic mobile devices and new information requirements including recency testing data were adopted. The HIV CBS system could also be integrated with several reporting systems, however, use of parallel HIV reporting systems including paper based system (HIV CBS forms and registers), Electronic patient monitoring system (ePMS), DHIS-2 and the EHR system result in duplication of tasks. This is consistent with findings by Ogungbemi et al., 2012, who found that the use of multiple unlinked HIV databases to capture program monitoring data result in duplication of effort and poor resource use [26].

We found the HIV CBS system was acceptable as all healthcare workers expressed willingness to continue participating in the system using the EHR system because it presents opportunities for reduced paperwork, secure storage of records and simple report generation. Our findings are consistent with Okeafor et al., 2017 where an evaluation of the HIV CBS system in Rivers State Nigeria revealed that all stakeholders were willing to continue to participate in the surveillance system [21]. 
The majority of healthcare workers had good knowledge of the HIV CBS system. This may be because health workers have a central role in HIV interventions which has enhanced their knowledge on the HIV CBS system. Data was analyzed at facility level and the majority of healthcare workers found the system to be useful to their daily operations. Data from the HIV CBS system was used to identify hotspots for targeted interventions, to identify risky behavior for communication strategies, drugs and HIV test kits supply management and to identify challenges. This is consistent with Nsubuga et al., 2020 who found that the system was utilized at facility level to analyze client data, and enabled staff to accurately order the exact number of antiretroviral drug for their clients [19]. Public health actions included health education, community outreach and awareness campaigns, enhanced contact tracing and HIV testing as well as distribution of condoms. However, there was no record of how the surveillance system was used to evaluate the success of the reported public health interventions.

\section{Study Limitations}

We could not objectively assess data quality of the HIV CBS system as there was no documentation of trainings, support and supervision visits thus we had to rely on information from health workers which was subject to recall bias.

\section{Conclusions}

The reasons for under-reporting of HIV cases through the EHR system were mainly the lack of reporting guidelines, limited coordination between units, insufficient knowledge of the EHR system and limited feedback from higher levels. We concluded that the overall health worker knowledge levels of the HIV CBS system were satisfactory. The HIV case-based surveillance system with recency testing performs well in terms of timeliness, flexibility and acceptability with high data quality however representativeness, stability and simplicity were not satisfactory. There were no SOPs for backup operations during system interruptions and health workers needed further training on the use of the EHR system including entering HIV retests, pediatric ART formulations, editing electronic health records and generating reports. The system was useful as the information was used to manage stocks and identify hotspots for new infections.

We recommended on-job refresher training on the EHR system for healthcare workers and data entry clerks to address identified training gaps as well as data analysis training. Support and supervision visits from higher levels and technical staff are critical. Healthcare workers also need to be sensitized and trained when new versions of the EHR system are released and installed. We also recommend the distribution of WHO clinical staging guidelines for HIV disease, the servicing of generators for backup electricity as well as the installation of solar power. We photocopied and distributed WHO clinical staging guidelines for HIV disease to three facilities. We also gave health education to health workers on the importance of reporting sentinel events for HIV patients. Health workers received 
further on-job training on the EHR system.

\section{Acknowledgements}

We would like to acknowledge the provincial medical directorate Manicaland Province, Mutare City Health Department, Department of Primary Health Care Sciences, University of Zimbabwe and Health Studies Office for all the help they rendered. Many thanks go to health workers in Mutare district for their participation.

\section{Conflicts of Interest}

The authors declare no conflicts of interest regarding the publication of this paper.

\section{References}

[1] Joint United Nations Programme on HIV/AIDS (2019) Global Factsheet for HIV/AIDS Estimates. http://aidsinfo.unaids.org

[2] World Health Organisation (2018) HIV/AIDS. WHO, Regional Office for Africa. https://www.afro.who.int/health-topics/hivaids

[3] HIV and AIDS in Zimbabwe (2015) Avert. https://www.avert.org/professionals/hiv-around-world/sub-saharan-africa/zimbabwe

[4] WHO_Consolidated_Guidelines_Annexes_3.5.2.pdf. https://www.who.int/hiv/pub/guidelines/WHO Consolidated Guidelines Annexes 3.5.2.pdf?ua $=1$

[5] PEPFAR (2019) HIV Case-Based Surveillance System with Biometric Code and Patient Linkage and Retention Tool. PEPFAR Solutions Platform (BETA).

https://www.pepfarsolutions.org/tools-2/2019/8/5/hiv-case-based-surveillance-syste m-with-biometric-code-and-patient-linkage-and-retention-tool

[6] Holmes, J.R., Dinh, T.-H., Farach, N., Manders, E.-J., Kariuki, J., Rosen, D.H., et al. (2019) Status of HIV Case-Based Surveillance Implementation-39 U.S. Pepfar-Supported Countries, May-July 2019. Morbidity and Mortality Weekly Report, 68, 1089. https://doi.org/10.15585/mmwr.mm6847a2

[7] Delcher, C. (2020) Perspective Piece Haiti's HIV Surveillance System: Past, Present, and Future. The American Journal of Tropical Medicine and Hygiene, 103, 1372-1375. https://doi.org/10.4269/ajtmh.20-0004

[8] Zimbabwe HIV Patient Monitoring and Case-Based Surveillance-Leveraging on Data to End AIDS by 2030. WHO, Regional Office for Africa.

https://www.afro.who.int/news/zimbabwe-hiv-patient-monitoring-and-case-basedsurveillance-leveraging-data-end-aids-2030

[9] Harklerode, R., Schwarcz, S., Hargreaves, J., Boulle, A., Todd, J., Xueref, S., et al. (2017) Feasibility of Establishing HIV Case-Based Surveillance to Measure Progress along the Health Sector Cascade: Situational Assessments in Tanzania, South Africa, and Kenya. JMIR Public Health and Surveillance, 3, e44. https://doi.org/10.2196/publichealth.7610

[10] Medical Research Council of Zimbabwe (2019) MRCZ Revised Zimbabwe Protocol of Surveillance of Newly Diagnosed HIV Cases. V1.6_03282019.

[11] CDC (2020) Strategic Information. Zimbabwe. CDC Global Health. 
https://www.cdc.gov/globalhealth/countries/zimbabwe/annual-report/strategic-info rmation.html

[12] Kim, A.A., Behel, S., Northbrook, S. and Parekh, B.S. (2019) Tracking with Recency Assays to Control the Epidemic: Real-Time HIV Surveillance and Public Health Response. AIDS, 33, 1527-1529. https://doi.org/10.1097/QAD.0000000000002239

[13] CDC (2001) Updated Guidelines for Evaluating Public Health Surveillance Systems. https://www.cdc.gov/mmwr/preview/mmwrhtml/rr5013a1.htm

[14] District-Population-Projection-Report-Manicaland. https://www.zimstat.co.zw/wp-content/uploads/publications/Population/populatio n/District-Projections/District-Population-Projection-Report-Manicaland.pdf

[15] Hall, H.I., Song, R., Gerstle, J.E. and Lee, L.M. (2006) Assessing the Completeness of Reporting of Human Immunodeficiency Virus Diagnoses in 2002-2003: Capture-Recapture Methods. The American Journal of Epidemiology, 164, 391-397. https://doi.org/10.1093/aje/kwj216

[16] Brown, S. (2010) Likert Scale Examples for Surveys. Iowa State University Extension.

https://www.extension.iastate.edu/Documents/ANR/LikertScaleExamplesforSurveys.pdf

[17] Turnberg, W., Daniell, W. and Duchin, J. (2010) Notifiable Infectious Disease Reporting Awareness among Physicians and Registered Nurses in Primary Care and Emergency Department Settings. American Journal of Infection Control, 38, 410-412. https://doi.org/10.1016/j.ajic.2009.07.013

[18] Gesulga, J.M., Berjame, A., Moquiala, K.S. and Galido, A. (2017) Barriers to Electronic Health Record System Implementation and Information Systems Resources: A Structured Review. Procedia Computer Science, 124, 544-551.

https://doi.org/10.1016/j.procs.2017.12.188

[19] Nsubuga, P., Mabaya, S., Apollo, T., Sithole, N., Komtenza, B., Matare, T., et al. (2020) Evaluation of the Zimbabwe HIV Case Surveillance Pilot Program, 2019. https://doi.org/10.2196/preprints.20034

[20] Gortakowski, H.W., Braunstein, S.L. and Shepard, C.W. (2010) Evaluation of the New York City HIV/AIDS Surveillance System.

[21] Okeafor, I.N. (2017) Evaluation of HIV Surveillance System in Rivers State, Nigeria. Nigerian Health Journal, 17.

https://www.ajol.info/index.php/nhj/article/view/154265

[22] Ezeudu, C., Nguku, P., Oladimeji, A. and Fawole, O. (2016) Evaluation of HIV/AIDS Surveillance System (2010-2013) in Enugu State April 2014. Online Journal of Public Health Informatics, 8. https://doi.org/10.5210/ojphi.v8i1.6525

[23] Naqibullah, H. (2020) Evaluation of HIV \& AIDS Surveillance System in Afghanistan. Journal of HIV and AIDS, 6. https://doi.org/10.16966/2380-5536.172

[24] Ngugi, B.K., Harrington, B., Porcher, E.N. and Wamai, R.G. (2019) Data Quality Shortcomings with the US HIV/AIDS Surveillance System. Health Informatics Journal, 25, 304-314. https://doi.org/10.1177/1460458217706183

[25] Sukums, F., Mensah, N., Mpembeni, R., Kaltschmidt, J., Haefeli, W.E. and Blank, A. (2014) Health Workers' Knowledge of and Attitudes towards Computer Applications in Rural African Health Facilities. Global Health Action, 7, 24534. https://doi.org/10.3402/gha.v7.24534

[26] Ogungbemi, K., Oyediran, K., Mullen, S., LaFond, A., Aderemi, A., Boone, D., et al. (2012) Using UN-AIDS's Organizing Framework to Assess Nigeria's National HIV Monitoring and Evaluation System. Open Journal of Preventive Medicine, 2, 372-378. https://doi.org/10.4236/ojpm.2012.23054 